\title{
Pediatric pain management competencies taught to nursing students in Rwanda: Perspectives of nurse educators, preceptors and nursing students
}

\author{
Philomene Uwimana*1, Donatilla Mukamana ${ }^{1}$, Oluyinka Adejumo ${ }^{1}$, Yolanda Babenko-Mould ${ }^{2}$ \\ ${ }^{1}$ School of Nursing and Midwifery, University of Rwanda, Rwanda \\ ${ }^{2}$ Arthur Labatt Family Shool of Nursing, Western University, Canada
}

Received: May 19, 2020

Accepted: August 17, 2020

Online Published: August 24, 2020

DOI: $10.5430 /$ jnep.v10n12p21

URL: https://doi.org/10.5430/jnep.v10n12p21

\begin{abstract}
Competency for pediatric pain management is fundamental for nurses' responsibility in caring for pediatric patients with pain. However, effective nursing management of pain in hospitalized children continues to be a challenge more often linked to competency deficit as a consequence of unpreparedness in the pre-licensing education. Previous studies have established that nursing students exhibited lack of knowledge and poor attitudes regarding pediatric pain management, but none of the studies were done in the Rwandan context. The current study explores the pediatric pain management competencies taught to nursing students in Rwanda. An exploratory descriptive qualitative design based on face-to-face individual interviews and focus group discussions was utilized. Fourteen nurse educators and preceptors and nineteen nursing students were recruited from five study settings to explore their perspectives about pediatric pain management competencies taught to nursing students. Participants' narratives were analysed using thematic analysis from which six main themes emerged. Participants narrated that competencies related to children pain assessment, pain medication and non-drug pain management interventions were taught to students. However, findings also revealed the challenges that impacted the teaching and learning of paediatric pain management, which need to be addressed for the improvement of pre-service training about pain management in children. The findings from the study suggested further research for a better understanding of the nature of those challenges to inform tailored strategies aimed at improving quality health care provision to children through an improved pediatric pain management education at the undergraduate level.
\end{abstract}

Key Words: Pediatric pain management, Competency, Nurse educator, Preceptor, Nursing student

\section{BACKGROUND}

Pain in children is a significant global public health concern. ${ }^{[1]}$ Evidence shows that unrelieved or under-managed pain has many implications such as delay in recovery, unnecessary suffering that often results in chronic pain, ${ }^{[2-4]}$ unwanted effects on the psycho-social development of the child, and the spiritual and financial state of the entire family. ${ }^{[5,6]}$ Children's ability to express their feelings about pain is dependent on their developmental level, and the nature of their prior pain experiences. ${ }^{[7,8]}$ As stated by the International Association for the Study of Pain (IASP), ${ }^{[9]}$ nurses play a critical role in caring for children with pain as they spend more time with the children and their families than other healthcare professionals. As such, nurses are key to assessing pediatric pain and communicating their findings to the care-team members for effective pain management

* Correspondence: Philomene Uwimana; Email: uwimphilo@yahoo.fr; Address: School of Nursing and Midwifery, University of Rwanda, Rwanda. 
in children. ${ }^{[10]}$ Effective relief of pain is the right of every child. ${ }^{[11]}$ King and Fraser $(2013$, p.2) posited that a concerted effort is required "... to place untreated pain at the top of the list of global health priorities" and that "this effort must attend to the complexity of pain and promote multimodal, multidisciplinary pain management from the outset". [12]

Nurse educators and preceptors are required to possess knowledge about and skills in pediatric pain management, as they strive to contribute to quality healthcare by facilitating students to acquire and utilize competencies for managing pain in children. Lasch et al. (2002, p.64) ${ }^{[13]}$ reported that "knowledgeable or pain conscious" faculty members and clinical mentors were an important source for students' exposure to pain management principles and influenced the quantity and quality of information students received. However, in a study about knowledge and attitudes of pain management among nursing faculty $(\mathrm{n}=96)$, Voshall et al. $(2013)^{[14]}$ found a lack of knowledge among faculty even though they had been exposed to varing levels of learning about pain management in their basic education. Less than half (37\%) of faculty felt effectively knowledgeable or prepared. Likewise, nursing students nearing graduation exhibited a deficit in knowledge and poor attitudes toward pediatric pain management. ${ }^{[7,15]}$ Such results are very concerning given that nursing students who do not have a strong degree of preparation about pediatric pain management during their pre-service education will likely practice nursing with limited competency about pain management in general and pediatric pain management in particular. Such circumstances will ultimately impact the quality of care provided to pediatric patients when it comes to pain management.

The aim was to explore nurse educators', preceptors', and nursing students' perceptions about and competency for pediatric pain management in the context of nursing education in Rwanda.

\section{Methods}

\subsection{Study design}

The study adopted an exploratory descriptive qualitative design, ${ }^{[16]}$ based on face-to-face individual interviews with nurse educators and preceptors, and focus group discussions with nursing students. Polit and Beck (2017) ${ }^{[17]}$ stated that in a qualitative descriptive study or "naturalistic inquiry", researchers collect data in a naturalistic worldview, which allows them to obtain broad insights and rich information from participants on the subject being studied. Hence, the researchers in this study sought to provide a comprehensive description from participants' perspectives about pediatric pain management competency acquisition in relation to nursing education in Rwanda.

\subsection{Ethical considerations}

The University of Rwanda, College of Medicine and Health Sciences Institutional Review Board provided ethics approval for this study with reference number 341/CMHS IRB/2018. Permission to collect data was granted from each study site and signed consent to participate was obtained from all the participants. Participation was voluntary.

\subsection{Study setting and participants}

The study was conducted in Rwanda at five sites. Academic settings included two nursing schools, of which one was a public institution that offers both a diploma and a baccalaureate degree in nursing, while another was a private nursing school offering a diploma program in nursing. Practice settings included one university teaching hospital, one referral hospital, and one district hospital.

The participants included nurse educators, preceptors, and senior-level nursing students in both diploma and baccalaureate programs. Participants were recruited using a purposive sampling technique so that the researcher could gain a broader understanding of the research subject from those who experienced it. ${ }^{[16,18]}$ Participant inclusion criteria involved being a nurse educator (faculty or clinical instructor) or preceptor (clinical nurse), having facilitated nursing students to learn about pediatric pain management in the classroom, in simulation and/or in the practice setting, and consenting to share one's experience of facilitating students' learning. Final-year nursing students were eligible to participate in the study as they had completed a course about pediatric nursing in which the component of pediatric pain management was taught.

A purposive sample of 14 nurse educators and preceptors were selected for individual in-depth interviews. This sample size was in keeping with what authors using interpretive methodology have recommended to support sufficient and rich data. ${ }^{[18,19]}$ Concerning student participants and the focus group approach to data collection, group dynamics of a focus group allows researchers to generate deep and rich data as participants share ideas and feelings that they may have about certain issues, but also, differences in perceptive between groups of individuals can be clarified. ${ }^{[20]}$ In this study, twenty nursing students were selected for focus group interviews.

In the process of purposively sampling participants, the primary researcher received permission from academic Principals and Deans to contact nurse educators via email to solicit their participation in the study, while the heads of academic nursing departments were contacted to request permission for the recruitment of students. The email that was sent by 
the primary researcher clearly stated that participation in the study was on a voluntary basis, and a letter of information and consent form were attached to the email. The primary researcher also liaised with the Matrons in the pediatric department of hospitals so that they could facilitate contact with preceptors in pediatric units. The Matrons held a meeting with individual preceptors to explain the study. Preceptors who expressed an interest in gaining more information about the study were informed to contact the primary researcher, as noted in the study letter of information. Those who contacted the primary researcher about the study first had any outstanding study questions addressed and then if they wished to participate in the study, an interview or focus group date time, and location were arranged.

\subsection{Data Collection Procedure}

A semi-structured interview guide was used to collect the data from nurse educators and preceptors. The interview guides had been developed based on expert opinion and relevant literature and included questions and probes related to pediatric pain management competency and about the educational preparation of nursing students in Rwanda.

Based on the choice of the interviewee(s), the individual interviews or focus groups were conducted either in English or Kinyarwanda. With permission of the participants, the responses were audio-recorded. Individual interviews took place in a quiet private room at the convenience of the participants, either in the nurse educator's office at a nursing school or at the hospital in a room allocated by the unit manager to support participant privacy. The length of the interviews ranged between 30 to 60 minutes.

Focus group discussions (FGD) were carried out with nursing students about the competencies for pediatric pain management they acquired through course work that was facilitated by nurse educators and preceptors. The FGDs were organised to take place at nursing schools and practice placement settings using an interview guide specific for the nursing student participants. The participants consented to be audiorecorded. Only nursing student participants and the primary researcher were in the room during the FGDs to support an environment conducive to open discussion. The FGDs averaged 60 minutes in length. Overall, the data collection process occurred across three months with nurse educators, preceptors, and students.

\subsection{Trustworthiness}

To ensure the scientific integrity of the research, ${ }^{[21]}$ four constructs of trustworthiness, namely credibility, transferability, dependability, and confirmability were maintained. Credibility was ensured by recruiting participants who met

Published by Sciedu Press the inclusion criteria before confirming their involvement in the study. Also, all researchers were engaged in the process of data analysis and met regularly to discuss and build a consensus on the emerging themes. Confirmability and credibility of the findings were supported by ensuring that the data accurately represented the information provided by participants, and this was consistently reviewed by senior researchers among the team. Anonymity and confidentiality of the participants were maintained through interview location and data security measures. A general description of the study setting and characteristics of the participants are noted to support transferability. In this study, the participants are referred to by using numbers as a means to support their anonymity. To ensure dependability, with the assistance of senior researchers and peers, an internal audit was done to check the consistency of the study process.

\subsection{Data Analysis}

The primary researcher listened carefully to the audiorecorded data and transcribed them verbatim. When participants shared experiences during interviews or FGDs in Kinyarwanda, that data was first transcribed into Kinyarwanda and then translated into English and re-checked by an expert translator. The data were analysed using thematic analysis steps as described by Braun and Clarke (2006), ${ }^{[22]}$ and Nowell et al. (2017). ${ }^{[23]}$ After multiple readings of the transcripts, each research team member analyzed the same transcripts separately and then all research team members discussed the emerging themes. This process supported establishing consensus through the coding process to thematic identification. NVivo 12 software was used for data management. ${ }^{[24]}$ The line-by-line coding technique was adopted to code the interviews using an interactive process, which generated initial codes. This initial coding was followed by grouping the coded smaller segments into larger segments that enabled the conceptualization of themes. Senior qualitative researchers reviewed the codebooks independently to ensure the coding was completed in keeping with the thematic analysis process. The use of thematic analysis permitted the researchers to ultimately illuminate themes related to nurse educators', preceptors', and nursing students' experiences of how pediatric pain management competencies were facilitated for nursing students in their diploma and baccalaureate nursing education programs. Data analysis concluded when participants' data did not reveal additional new themes or sub-themes.

\section{Findings}

\subsection{Description of Participants}

Fourteen nurse educators and preceptors consented to participate (six nurse educators and eight preceptors out of a population of 16 in total) in the study. Over $75 \%$ of these 
participants were over 31 years of age, with the highest level of education attained being a baccalaureate degree in nursing, and $57 \%$ were female. Nurse educators and preceptors reported having between 1 to 2 years of prior experience in the role of teaching about pediatric pain management. The majority of nurse educators had not received any formal education about pediatric pain management apart from what they had originally learned in their nursing education program when they were students.

Two focus group discussions were held with nineteen nursing students from two sites. Nine students were in Year 3 of their program, while 10 students were in Year 4. The majority of participants in the focus groups were male and their mean age was 24 years.

\subsection{Thematic analysis}

Six main themes emerged from the individual interviews and the FGDs. The first five themes, each one with sub-themes, correspond to the categories of pediatric pain management competencies. In particular, those related to understanding pain, its assessment, and management in children, communication skills, and collaborative practice. The sixth theme was related to the challenges that impact the teaching and learning of pediatric pain management.

\subsubsection{Understanding pain}

Some participants stated that the first thing they do to support students' acquisition of pediatric pain management competency is to facilitate the development of an understanding of the nature of pain and the complexities of pain management that must be considered when caring for children. Nurse educators and preceptors shared that one of their priorities for teaching pediatric pain management was to “...teach them what pain is - its pathophysiology .... we discuss pain in theory... it's also critical to know the cause of the pain because you can't only treat the pain without even knowing first its cause." (Nurse Educator1)

Nurse educators and preceptors reported that once students displayed evidence of having developed foundational knowledge about pain, the next phase of teaching would be to facilitate their understanding of pediatric pain in particular. A couple of educators voiced: “.... normally the way we facilitate students, we start by explaining what pain is and let them understand that children also experience the pain, that it is even worse than adults." (Nurse Educator2)

“... it becomes very unique to children because they feel much the pain..., it is important that nursing students whom we facilitate competency acquisition consider pain as a serious thing they should take into consideration so that they can provide adequate care to children." (Nurse Educator3)
This was confirmed by a nursing student participant in FGD who stated, "... we have learned about what is pain in children, how it looks like..." (Student1)

\subsubsection{Assessment of pediatric pain}

From the perspectives of nurse educators, preceptors, and nursing students, pediatric pain assessment was taught to students using a range of techniques. Participants mentioned that nursing students learned how to assess pain in children by observing pain measurements/scores from different pain scales (e.g Faces scales, Neonatal Infant Pain Scale, FLACC, Numeric Rating Scale). A student said regarding the use of pain scale, “... we have learned that pediatric age is from an infant to 18 years old. ... they express pain in different ways. That is why we use different pain scales in assessing their pain .... We have learned also how to choose a good pain scale.” (Student2)

In the practice setting, educators and preceptors supported students' competency acquisition by teaching them about how to assess pediatric pain by observing the child's facial expression and changes in their behaviour such as not feeding effectively, as well as nuanced changes in vital signs.

".... we also show to the students the parameters of the child's vital signs. We tell the students, 'You know that generally, a child in this age his/her heart rate ranges between this figure and this, if you see that the heart rate of the child is beyond the normal range, you can think to check the pain, the fever." (Preceptor1)

Nursing students expressed how they were involved in a nonaided observation technique for pediatric pain assessment. "We learned different ways to assess a child with pain. observing that child is crying differently from how he used to do, the child may be screaming or may seem to isolate him/herself. You may also find that the child is not breastfeeding well, the arms are extended or abnormally flexed." (Student3)

\subsubsection{Management of pediatric pain}

For the management of pain, educators and preceptors, as well as students distinguished modes of treating pain using pharmacological agents that may vary from non-opioid analgesia to powerful opioid pain medication with reference to the WHO analgesic ladder, and non-pharmacological interventions for pediatric pain management. They further mentioned that students were guided to know the classification of pain medications and their use in managing pain according to the type or severity of pain. A preceptor noted that: "... we show the students which medication - You can start with a non-steroidal analgesia and after the time it is expected that pain will have reduced, if the pain was not reduced or the child is having more pain, you can give 
another type of painkiller.” (Preceptor1)

Additionally, some nursing student participants mentioned that they also learned how to assess for and manage adverse events resulting from the administration of pain medication to children, and to evaluate the outcome of the treatment of the event. Some participants revealed that students were taught that when a pediatric patient is assessed with mild pain, they should use non-pharmacologic techniques to relieve the pain instead of using medication.

"We teach students that, ... if a child has mild pain, they can start with those non-pharmacological strategies like distracting the child with toys, to ask the parent to be nearby the child, changing child's position --in order to reduce the pain of the child with non-pharmacological interventions,..." (Preceptor2)

This was corresponded by nursing students who noted that “...non-pharmacological ways of pain management, we learned that ...to ask his/her mother to breastfeed. Also, they taught us that we can give something which is delicious and place it in the child's mouth." (Student4)

\subsubsection{Communication skills}

Often children with pain may not be able to describe clearly what they are feeling to be a pain, especially when they are still very young or due to other developmental factors. As such, nurse educators and preceptors indicated that nursing students needed skills that would enable them to interact with children experiencing pain as well as their parents/caregivers Effective communication with pediatric patients and their families/caregivers is a competency that is developed through ongoing learning and practice. As a nurse educator participant stated, “... students also learn to talk and communicate with parents/caregivers as we do." (Nurse Educator2)

And a student noted, "I used the gained knowledge to care for the child, trying to talk to her/him, comforting her/him and distracting her/him.” (Student2)

\subsubsection{Collaborative practice}

Some participants confirmed that nursing students learn about teamwork and interprofessional collaboration in the context of pediatric pain management. Facilitators and learners concurred that the involvement of the parents/caregivers, nurses, and physicians is important and discussed during students' course work about pediatric nursing theory and in practice, as it's a key to supporting the pain management process. Students are initiated to a culture of teamwork with all stakeholders involved in the care of the child. As a student stated, “......we [students] talk with nurses and doctors about the methods and the pain medications they use, we tell them what we have studied ... if they say that it can be done according to the source and protocol they have, it is applied and they teach us their methods of managing the pain, we share ideas.” (Student5)

Also, students are taught to seek the input of the family in the care of the child. As a nurse educator noted:

"In clinical mainly in pediatric unit with my student when we meet a pediatric patient with pain, I tell them an assessment should be done, you have to involve your patient, involve next of kin, parents, the family and then after, you can find what's wrong on the patient." (Nurse Educator4)

\subsubsection{Challenges impacting the teaching and learning of pediatric pain management competencies}

Study participants, primarily students, discussed some concerns about issues around facilitating nursing student competencies for pediatric pain management. Those concerns included the challenges related to substandard teaching plans, ineffective role modeling in the practice setting, perceived negligence of pain in children, and knowledge and skill deficiency among educators and preceptors.

\section{Substandard teaching plans}

The short time allocated to the topic of pain management in the module of pediatric nursing and the insufficient practice in skills lab were mentioned as hindrances for students to master the fundamentals of pediatric pain management. Regarding practicing pediatric pain management in the skills lab, few nurse educators seemed to think that it was not feasible to teach pediatric pain management in the simulation setting, mainly because it might require high-fidelity pediatric simulation equipment that is not available. One educator stated that students were facilitated in skills lab to acquire nursing skills other than those relating to pediatric pain management.: "In the skills lab nothing is done related to pain management - nothing, when we go there it is for something else. So, what I can say is that their practice is low." (Nurse educator1)

\section{Ineffective role modeling in practice}

This was mentioned as a challenge for students in some practice settings. Ineffective role modeling of pain management practices and poor attitudes about pediatric pain management among a few nurses was witnessed in clinical practice. Student participants also described a lack of clarity about how and what to report around pediatric pain and lack of guidance on pediatric pain management. Students associated such experiences with perceptions of having had unsatisfactory mentorship or learning opportunities to support knowledge and skill development. Even though these issues concern the unprofessional behavior of individual nurses and not the health system in general, they negatively impact students' 
learning about their ability to assess and manage pediatric pain. Normally, the nurse should follow the guidelines and protocols in place on the assessment and relief of pain in children and document the findings and interventions. For instance, where a child with severe pain requires opioid medication for relief, the nurse is expected to administer the medication following the physician's prescription in which medication dose and time for administration are specified. In another instance, a nursing student during the clinical placements witnessed the malpractice of one nurse regarding pediatric pain management. The student shared his/her experience, “... the nurse told me that there is nothing to do for the child. 's/he cries all the time... ok, look if you can find morphine and give it to him/her', the nurse said. It was a young child, the nurse did not tell me which dose of morphine to give to the child, did not know if I will find morphine, s/he put me in a confusing situation, I did not know for the child of that age how much of morphine to give it was not written in the patient's file. ... this was a big challenge because what we learned at school we miss the opportunity to put it into practice during the clinical placement.... I don't know how else to say it." (Student6).

\section{Perceived negligence of pain in children}

A large proportion of student participants perceived that in practice settings, pain in children was considered to be neglected and most of the time they [students] missed opportunities to learn or were learning an ineffective approach to pain management, which could ultimately translate to unmet patient care needs. For example, the participants mentioned that some nurses in practice settings administer weak painkillers to suffering children and encourage children to bear with the pain because they fear to administer morphine. Furthermore, the participants explained that to some extent, the suffering child would be required to hide the pain and pretend that she/he is all right because, in the Rwandan culture, people tend to value stoisim and prefer to hide their suffering. Children are often encouraged to remain strong 'like a man', otherwise, it is a sign of cowardice. As a student stated:

“... where I have been for clinical placement, nurses waited until the child cries due to pain; often it became severe pain. When a child cries because of pain and the assessment wasn't well done to manage the pain, they told the child to stay strong, not to cry.” (Student1)

\section{Knowledge and skill deficiency among the facilitators}

The participants revealed that nurse educators and/or nurse preceptors have limited knowledge and skill about effective pediatric pain management probably because they did not benefit from training on that aspect of the practice. For the participants, some facilitators (nurse educators or preceptors) might not have had the opportunity to update their knowledge for pain management since their basic nursing education as stated by one of them: "my colleagues..., there are many who did not get the opportunity to learn about pain in children or pain management in general. Thus, it is a challenge, if $s /$ he did not receive training on that, s/he will not give anything to students because it is like they are at the same level up to now." (Nurse Educator5)

The fact that preceptors might not be well equipped in pediatric pain management negatively impacts the student learning process. Some of the students said: "people we find in clinical placement, to my view don't have yet the skills to manage pain in children.” (Student7)

“... you understand that in some institutions also nurses are not able to practice effective pain management; they are not able to assess and manage pain.” (Student5).

\section{Discussion}

The findings from the current study indicate that nurse educators and preceptors expect that nursing students will develop a broad understanding of pain pathophysiology, children's psychological development, and pain perceptions as well as related complications or sequelae to children when pain is unrelieved. These findings are consistent with previous scientific work ${ }^{[5,11,25,26]}$ and show the importance for future nursing professionals of being cognisant about the phenomenon of pain in children for its effective assessment and management.

The data highlight that nursing students are taught how to use a variety of techniques for assessing pain in children such as observing the behaviour of the child, assessing the vital signs, and using appropriate pain assessment scales, together with reports from patients or parents/caregivers on the onset, the duration, the intensity and the location of the pain. Similar findings regarding required competencies for pain assessment were found in previous studies that aimed to address pain management education in the prelicensing education of healthcare professionals. ${ }^{[11,25,27]}$ In the current study, evaluating vital signs was cited as a means to assess pain in children, which concurred with the findings from another study. ${ }^{[28]}$ Controversy appears with researchers who found that vital signs may not be reliable indicators for accurate pain assessment in children, ${ }^{[29,30]}$ as physiological changes may occur due to clinical condition that the child presents and not necessarily because of pain. That notwithstanding, nursing students should be educated to distinguish the variations in vital signs due to pain and the variations caused by other health conditions during the provision of 
care to pediatric patients.

Moreover, working collaboratively with healthcare professionals and caregivers including the parents and the child him/herself in assessing and alleviating patients' pain was viewed by the participants as another important factor that fosters students' learning about pediatric pain management. All this supports what was stated by Fishman et al. $(2013)^{[25]}$ in connection with interprofessional education for pain management. When nursing students are integrated into the interprofessional team for the management of pain in children, they communicate easier with each member regarding any aspect of pain management of the pediatric patient. Students become responsible by taking part in their learning process. Findings from this study suggest that nursing students are facilitated to acquire communication skills that are needed for effective pediatric pain management, which is similar to a report from previous work. ${ }^{[26]}$

Concerning pain management in children, the findings from this study indicated that nursing students were taught the use of medications and non-pharmacologic interventions to relieve pain in children, the WHO analgesic ladder, use of multimodal analgesia along with assessing pain medication side effects, and evaluation of treatment outcome, standing in agreement with scientific works on pain management education. ${ }^{[11,27]}$ However, the translation into practice of what is taught and/ or retained regarding pediatric pain management may be highly variable considering the challenges relating to pediatric pain relief practices raised by participants of this study. Yet, national guidelines and protocols on pain management exist as a reference for educators in prelicensing pain education and healthcare professionals in practice settings for effective pediatric pain management. Still, some nurse educators and nurse preceptors in the clinical setting experience difficulties to implement them. It may be interpreted as the fact that nurse educators and preceptors in the study were aware of the pain guidelines and protocols, but were not given proper guidance on their implementation during continuing education on the management of pediatric pain as it was expressed by the majority of them. A previous study on knowledge and practice of immediate post-operative pain management among nurses in Rwanda indicated that nurses had a high level of knowledge about immediate postoperative pain management while their level of practicing immediate post-operative pain management was moderate, ${ }^{[31]}$ which negatively impacted the learning of practical skills on pediatric pain management of nursing students during their clinical rotations. To address this problem, continuing professional education for nurse educators and clinical nurses in pediatric pain management should be considered.
Participants in this study argued that pediatric pain management is probably not seen as a priority given the short time allocated to it in teaching and the fact that students were not given the opportunity to practice it in skills lab for their preparation for clinical placement. This study reinforces the findings of other studies that indicated inadequate pain education in pre-licensing education. ${ }^{[32,33]}$ Nurse educators should consider employing innovative approaches suited to the context of limited resources and elaborate scenarios that can be utilized to teach nursing students the practice of pediatric pain management with the resources available in the skills labs. Also, in practice settings, the negligence of pain management in children and the myths around the use of morphine was identified by participants as major concerns. On one hand, this could be perceived as ethical issues around healing which are linked to the beliefs regarding pain in children and the fear of administering morphine. On the other hand, it could be a consequence of the cultural influence on the decision to manage pain or not. Similar to the current study, previous studies indicated that judgments and beliefs of nurses ${ }^{[33]}$ and fear of side effects of opioids ${ }^{[34,35]}$ were barriers to effective assessment and management of pain in children. The cultural effect is more sensitive in the Rwandan context whereby a child is encouraged to remain stoic and not to show or express pain s/he is feeling. "Kwihanaga" is a very common word in Kinyarwanda which may be nearly translated to "bear with it".

Ineffective role modeling was of concern for nursing students because it reinforced inappropriate clinical practices and attitudes as regards to pediatric pain management. This finding concurs with previous research. ${ }^{[36]}$ Nurses that appear to lack interest in what they do, could be linked with inadequate basic nursing education and/or to a more demanding workload. The lack of role modeling can impede students' acquisition of competency for pediatric pain management.

The majority of nurse educators and preceptors admitted that they did not receive specific training in pediatric pain management. This knowledge deficit and inappropriate attitudes among some nurses resulted in negligent response to pain in hospitalized children as reported by nursing students. This is similar to the findings from other studies that reported poor knowledge and attitudes toward pediatric pain management among nurses, ${ }^{[34,37]}$ and that nursing faculty who learned about pain management only in their pre-service training exhibited a knowledge deficit regarding pain management. ${ }^{[14,26,38,39]}$ In 2011, the national palliative care policy and the national training in palliative care for end-of-life program that included pain management were launched in Rwanda, ${ }^{[40]}$ opening access to training regarding pain management. Since then, some hospitals opted to train healthcare 
providers including nurses working at those settings on pain management, but not many of the health care providers were trained as it was mentioned by the participants of this study. Also, there was a turnover of those who were trained, while others were employed after the training was conducted. Furthermore, the in-service training on pain management was organized by hospitals and did not concern nurse educators. Therefore, more collaboration should be considered in the future between teaching institutions and clinical settings for conducting continuous education on pediatric pain management for nurse educators and nurse preceptors, aiming at improving pediatric pain management education in undergraduate nursing programs and promoting the optimal healthcare delivery to the suffering child.

\section{Conclusion}

Pediatric pain management competencies taught to nursing students in Rwanda seem to be congruent with the findings from previous studies in other countries. However, the participants pointed out the challenges that impact the way nursing students acquire competencies for managing pain in children. The findings from the current study suggest that there is a deficit in knowledge starting with nurse educators and nurse preceptors, then transferred to nursing students that will become nurse educators and preceptors in the future. Further exploration is important to resolve this deficit. Looking at the capacity (i.e. knowledge and attitudes) of nurse educators and preceptors and how they understand pediatric pain management is critical for resolving this issue. The study indicated other concerns raised by participants including substandard teaching plans, ineffective role modeling in the practice setting, and perceived negligence of pain in children. Continuous education on pediatric pain management conducted regularly may help to address those concerns. At nursing schools, educators should be innovative, using the available resources in teaching to students pediatric pain man- agement practice in skills lab before the practice rotations. Further studies need to focus on other factors that impede the teaching of pediatric pain management competencies to nursing students. From there, a clear understanding of the challenges would inform tailored strategies aimed at improving quality health care provision to children through an improved pediatric pain management education to nursing students.

\section{Strengths and limitations}

This study explored pediatric pain management competencies taught/learned in Rwanda and involved nurse educators, preceptors, and nursing students as the key stakeholders. The study participants were selected from various areas of practice such as a rural district hospital, referral, and university teaching hospital as well as from public and private nursing schools. Nevertheless, the study is limited by the fact that it was conducted in the Rwandan context only and may not be generalized to other contexts. Also, the findings relate only to what was reported by the participants. An added review of the syllabi in data collection could have provided further insights into what is meant to be taught or learned in the programs.

\section{ACKNOWLEDGEMENTS}

We acknowledge the support received from the University of Rwanda and Western University through the Training, Support, and Access Model for Maternal, Newborn, and Child Health in Rwanda (TSAM) Project. The research was financially supported by a scholarship provided to PU by a grant to Prof. David Cechetto, Western University, Canada, from the Government of Canada, provided through Global Affairs Canada. The scholarship mainly covered PU's tuition and fieldwork expenses in Rwanda.

\section{CONFLicts OF InTEREST Disclosure}

The authors declare that there is no conflict of interest.

\section{REFERENCES}

[1] World Health Organization. Persisting pain in children package: WHO guidelines on the pharmacological treatment of persisting pain in children with medical illnesses [Intrernet]. World Health Organization; 2012. Available from: https://apps.who.int/iri s/handle/10665/44540

[2] Batoz H, Semjen F, Bordes-Demolis M, et al. Chronic postsurgical pain in children: Prevalence and risk factors. A prospective observational study. Br J Anaesth. 2016; 117(4): 489-96. PMid:28077537 https://doi.org/10.1093/bja/aew260

[3] Kahsay H. Assessment and treatment of pain in pediatric patients. Curr Pediatr Res. 2017; 21(1): 148-157.

[4] Mathews L. Pain in Children: Neglected, Unaddressed and Mismanaged. Indian J Palliat Care. Jan 2011; 17(Suppl): S70-S73.
PMid:21811376 https : //doi.org/10.4103/0973-1075.7624 7

[5] Gadallah MAE, Hassan AM. Undergraduate nursing students ' knowledge and attitude regarding pain management of children in Upper Egypt. 2017; 7(6). https://doi .org/10.5430/jnep.v7n6p100

[6] Liossi C, Howard RF. Pediatric chronic pain: Biopsychosocial assessment and formulation. Pediatrics. 2016; 138(5). PMid:27940762 https://doi.org/10.1542/peds.2016-0331

[7] Ljusegren G, Johansson I, Gimbler BI, et al. Nurses' experiences of caring for children in pain. Child Care Health Dev. 2012; 38(4): 464-70. PMid:21671980 https://doi.org/10.1111/j.1365-2 214.2011.01262.x

[8] Noel M, Chambers CT, McGrath PJ, et al. The influence of children's pain memories on subsequent pain experience. Pain [Internet]. 2012; 
153(8): 1563-72. PMid:22560288 https://doi.org/10.1016/j. pain.2012.02.020

[9] International Association for Study of Pain. Curriculum Outline on Pain for Nursing [internet]. IASP, Washington, DC:, 2018 [cited 2020 March 02]. Available from: https://www.iasp-pain.org/Educ ation/CurriculumDetail . aspx? ItemNumber $=2052$

[10] Marshall C, Mscn RN, Forgeron P, et al. Exploration of nurses' pediatric pain management experiences in rural hospitals: A qualitative descriptive study. Appl Nurs Res [Internet]. 2018; 42(December 2017): 89-97. PMid:30029720 https ://doi .org/10.1016/j . ap $\mathrm{nr} .2018 .06 .009$

[11] Olmstead DL, Scott SD, Austin WJ. Unresolved pain in children: A relational ethics perspective. Nurs Ethics. 2010; 17(6): 695-704. PMid:21097968 https://doi.org/10.1177/09697330103789 32

[12] King NB, Fraser V. Untreated Pain, Narcotics Regulation, and Global Health Ideologies. PLoS Med. 2013; 10(4): 2-5. PMid:23565063 https://doi.org/10.1371/journal.pmed.1001411

[13] Lasch K, Greenhill A, Wilkes G, et al. Why Study Pain? A Qualitative Analysis of Medical and Nursing Faculty and Students' Knowledge of and Attitudes to Cancer Pain Management. J Pal Med. 2002; 5(1): 57-71. PMid:11839228 https://doi.org/10.1089/1096 6210252785024

[14] Voshall B, Dunn KS, Shelestak D. Knowledge and attitudes of pain management among nursing faculty. Pain Manag Nurs. 2013; 14(4). PMid:24315276 https://doi.org/10.1016/j.pmn.20 12.02 .001

[15] Omari OAl. Knowledge and attitudes of Jordanian nursing students toward children' s pain assessment and management: A cross-sectional study. 2016; 6(3): 51-8. https://doi.org/10.5430/jnep.v6n $3 \mathrm{p} 51$

[16] Neergaard MA, Olesen F, Andersen RS, et al. Qualitative descriptionthe poor cousin of health research? BMC Med Res Methodol. 2009; 9(1). PMid:19607668 https ://doi .org/10.1186/1471-2 288-9-52

[17] Polit DF, Beck CT. Nursing research: generating and assessing evidence for nursing practice. 10th ed. Philadelphia: Wolters Kluwer; c2017.

[18] Creswell JW (University of Nebraska-Lincoln). Research design: qualitative, quantitative, and mixed methods approaches. 4th ed. Los Angeles: SAGE Publications; c2014.

[19] O'Reilly M, Parker N. "Unsatisfactory Saturation": A critical exploration of the notion of saturated sample sizes in qualitative research. Qual Res. 2013; 13(2): 190-7.https ://doi.org/10.1177/1468 794112446106

[20] Rabiee F. Focus-group interview and data analysis. Proc Nutr Soc. 2004; 63(4): 655-60. PMid:15831139 https ://doi .org/10.107 9/PNS2004399

[21] Elo S, Kaariainen M, Kanste O, et al. Qualitative Content Analysis: A Focus on Trustworthiness. SAGE Open. 2014 Feb; 4(1). https://doi.org/10.1177/2158244014522633

[22] Braun V, Clarke V. Using thematic analysis in psychology. Qualitative Research in Psychology. 2006; 3(2): 77-101. https ://doi .or g/10.1191/1478088706qp063oa

[23] Nowell LS, Norris JM, White DE, et al. Thematic Analysis: Striving to Meet the Trustworthiness Criteria. Int J Qual Methods. 2017; 16(1): 1-13. https://doi.org/10.1177/1609406917733847

[24] Zamawe FC. The implication of using NVivo software in qualitative data analysis: Evidence-based reflections. Malawi Med J. 2015; 27(1): 13-5. PMid:26137192 https://doi.org/10.4314/mm . v27i1.4
[25] Fishman SM, Young HM, Arwood EL, et al. Core Competencies for Pain Management: Results of an Interprofessional Consensus Summit. Pain Med. 2013; 14: 971-81. PMid:23577878 https://doi.org/10.1111/pme.12107

[26] Herr K, St. Marie B, Gordon DB, et al. An interprofessional consensus of core competencies for prelicensure education in pain management: Curriculum application for nursing. J Nurs Educ. 2015; 54(6) 317-27. PMid:26057425 https://doi.org/10.3928/01484834 $-20150515-02$

[27] Hurley-Wallace A, Wood C, Franck LS, et al. Paediatric pain education for health care professionals. Pain Reports. 2019; 4(1): 1-5. PMid:30801042 https://doi.org/10.1097/PR9.0000000000 000701

[28] Vieira V, Marques L, Mello D, et al. Evaluation of pain in the pediatric patient by nurse in the hospital. J Pediatr Intensive Care. 2012; 2: 87-93.

[29] Erden S, Demir N, Ugras GA, et al. Vital signs: Valid indicators to assess pain in intensive care unit patients? An observational, descriptive study. Nurs Heal Sci. 2018; 20(4): 502-8. PMid:30027620 https://doi.org/10.1111/nhs.12543

[30] Herr K, Coyne PJ, McCaffery M, et al. Pain Assessment in the Patient Unable to Self-Report: Position Statement with Clinical Practice Recommendations. Pain Manag Nurs [Internet]. 2011; 12(4): 230-50. PMid:22117755 https ://doi .org/10.1016/j.pmn. 20 11.10 .002

[31] Umuhoza O, Chironda G, Katende G, et al. Perceived knowledge and practices of nurses regarding immediate post-operative pain management in surgical wards in Rwanda. A descriptive cross-sectional study. Int J Africa Nurs Sci. 2019.

[32] Campbell E. Faculty Perspectives of Teaching Pain Management to Nursing Students. Pain Manag Nurs [Internet]. 2019. PMid:31492600 https://doi.org/10.1016/j.pmn.2019.07.010

[33] Carr ECJ, Briggs E V, Briggs M, et al. Understanding factors that facilitate the inclusion of pain education in undergraduate curricula: Perspectives from a UK survey. 2016.

[34] Twycross A. Nurses' Views About the Barriers and Facilitators to Effective Management of Pediatric Pain. Pain Manag Nurs [Internet]. 2013 Dec 1 [cited 2019 Nov 29]; 14(4): e164-72. PMid:24315269 https://doi.org/10.1016/j.pmn.2011.10.007

[35] Alotaibi K, Higgins I, Chan S. Nurses' Knowledge and Attitude toward Pediatric Pain Management: A Cross-Sectional Study. Pain Manag Nurs [Internet]. 2019; 20(2): 118-25. PMid:30528361 https : //doi.org/10.1016/j.pmn.2018.09.001

[36] Mackintosh-franklin C. Pain: A content review of undergraduate pre-registration nurse education in the United Kingdom. Nurs Educ Tod [Internet]. 2017; 48: 84-9. PMid:27723538 https://doi .or $\mathrm{g} / 10.1016 / \mathrm{j}$. nedt .2016 .08 .035

[37] Amponsah AK, Oduro E, Bam V, et al. Nursing students and nurses' knowledge and attitudes regarding children's pain: A comparative cross-sectional study. PLoS One. 2019; 14(10): 1-12. PMid:31600329 https://doi.org/10.1371/journal. pone.0 223730

[38] Duke G, Haas BK, Yarbrough S, et al. Pain Management Knowledge and Attitudes of Baccalaureate Nursing Students and Faculty. Pain Manag Nurs. 2013; 14(1): 11-9. PMid:23452522 https: //doi.org/10.1016/j.pmn.2010.03.006

[39] Watt-Watson J, Murinson BB. Current challenges in pain education. Pain Manag. 2013; 3(5): 351-7. PMid:24654868 https: //doi.org/10.2217/pmt.13.39

[40] Unknown. Rolling out Rwanda's national palliative care programme Bull World Health Organ. 2018 Nov 1; 96(11): 736-737. 